\title{
Role of 3D Echocardiography in Cardiac Surgery: Strengths and Limitations
}

\author{
Edwin Wilberforce Turton ${ }^{1}$ • Jörg Ender ${ }^{1}$
}

Published online: 21 July 2017

(C) The Author(s) 2017. This article is an open access publication

\begin{abstract}
Purpose of Review This review aims to highlight the general and specific strengths and limitations of intraoperative 3D echocardiography. This article explains the value of real-time three-dimensional transesophageal echocardiography (RT 3D TEE) during cardiac surgery and cardiac interventions.

Recent Findings Recently published recommendations and guidelines include the use of RT 3D TEE. RT 3 D TEE provides additional value particularly for guidance during cardiac interventions (i.e., transcatheter mitral valve repair, left atrial appendix and atrial septal defect closures), assessment of the mitral valve in surgical repair, measurement of left ventricular outflow tract area for transcatheter valvular replacements, and estimating right and left ventricular volumes and function. The exact localization of paravalvular leakage is another strength of RT 3D TEE. The major limitation is the reduced temporal resolution compared to 2D TEE. Summary Three-dimensional echocardiography is a powerful tool that improves communication and accurate measurements of cardiac structures.
\end{abstract}

This article is part of the Topical Collection on Cardiovascular Anesthesia

Jörg Ender

joerg.ender@medizin.uni-leipzig.de

Edwin Wilberforce Turton

edwin.turton@medizin.uni-leipzig.de

1 Department of Anesthesiology and Intensive Care Medicine, Heart Center Leipzig, Struempellstr 39, 04289 Leipzig, Germany
Keywords Three-dimensional echocardiography (3DE) $\cdot 3 \mathrm{D}$ transesophageal echocardiography (TEE) $\cdot 3 \mathrm{D}$ transthoracic echocardiography (TTE) · Intraoperative echocardiography · 3D data sets $\cdot 3 \mathrm{D}$ modes $\cdot$ Full volume $(\mathrm{FV}) \cdot$ Color flow Doppler (CFD) $\cdot$ Real time (RT) $\cdot$ Live $\cdot$ Zoom $\cdot$ Simultaneous multiplane/biplane/X-plane imaging · Cardiac surgery · Interventions $\cdot$ Transcatheter procedures . Echocardiographer · Spatial resolution · Temporal resolution . Guidelines and recommendations · Valvular surgery · Future developments $\cdot$ Strengths $\cdot$ Limitations

\section{Introduction}

Since the first use of transesophageal echocardiography (TEE) during cardiac surgery in 1979 [1], twodimensional (2D) TEE has become a class I indication for perioperative use in almost every major cardiac surgical procedure [2].

Three-dimensional transesophageal echocardiography (3D TEE) was first available as an offline model after acquisition of multiple 2D examinations [3] that were very time consuming and user unfriendly [4]. Real-time 3D transesophageal echocardiography (RT 3D TEE) using a matrix probe evolved during the late 2000s [5] and led to the widespread use of this technique in cardiac surgery today.

This article highlights the strengths and limitations of RT 3D TEE in daily clinical practice during cardiac surgery and interventional cardiac procedures. This article is divided into two parts. The first discusses the strengths and the limitations of 3D echocardiography in comparison to $2 \mathrm{D}$ echocardiography (2DE), and the second outlines the specifics of RT 3D TEE looking at different cardiac structures. 


\section{Cardiac Surgery and Three-Dimensional Echocardiography}

\section{General Strengths and Limitations}

Two-dimensional echocardiography to visualize the 3D structure of the heart requires cognitive reconstruction from multiple 2D images. This is no longer required in 3D echocardiography. Further, while 2D echocardiography only reveals crosssectional views of the heart, $3 \mathrm{D}$ echocardiography is more akin to a Cartesian coordinate system [6••], enabling us to display the anatomic view of the heart. This simplifies communication between physicians and allows for better orientation, which is particularly helpful for image-guided interventional procedures [2].

Because ultrasound propagation is equal in both 2D and 3D echocardiography, the use of more Cartesian coordinate-like data to improve spatial orientation leads to reduced temporal resolution. Practically, the echocardiographer using 3D echocardiography has to decide between the optimal temporal and spatial resolutions when imaging specific cardiac structures.

Both temporal and spatial resolutions play a role in the quality of images produced by $3 \mathrm{D}$ echocardiography. Images need to be of adequate quality to allow qualitative and quantitative assessment. Temporal resolution is displayed as volumes per time unit (volume rate), and this can be increased by decreasing the volume size (sector) imaged. Spatial resolution can be improved by reducing the volume acquired or by increasing the number of scan lines through the volume. Increasing the number of scan lines for a specific volume will reduce its temporal resolution.

Different 3D modalities are available (i.e., wide-sector zoom, narrow sector, full volume (FV) mode with and without color Doppler). The technical aspect of these different modalities will not be discussed in detail in this article, but it is explained elsewhere $[7 \bullet \bullet]$.
The inherent physical properties of ultrasound and with that the limitations of ultrasound imaging in general are equally applicable to 3D and 2D echocardiography. Additionally, there are specific 3D-based artifacts such as stitching artifacts, dropout, "blurring" or thickening, blooming, and rail road artifacts [8-10]. Dropout artifacts can mimic valvular perforations and periprosthetic leaks [11].

Another limitation comes with displaying 3D echocardiographic images on a 2D screen. In doing so, colors are used to give a sense of depth and reality [12••]. Therefore, accurate measurements cannot be made in RT 3D mode [13].

\section{Specific Strengths and Limitations (See Table 1)}

\section{Left Ventricle Volume and Function}

Simultaneous multiplane mode allows the recommended biplane measurement for ejection fraction of the same heartbeat, whereas in 2D TEE assessment, it can only be performed in two different heartbeats [14].

With FV mode and multiple beat acquisitions, the left ventricular volume and function can be more accurately determined by $3 \mathrm{D}$ than by $2 \mathrm{D}$ echocardiography when compared to other image modalities like multidetector computed tomography (MDCT) or cardiac magnetic resonance imaging (CMRI) [15••]. Three-dimensional echocardiography is more accurate than both contrast enhanced 2D echocardiography and 2D echocardiography alone in the measurement of LV ejection fraction, but still underestimates LV volumes compared to CMRI [16]. The strength of left ventricular assessment with $3 \mathrm{D}$ echocardiography is that no geometric assumption is necessary and that it is unaffected by foreshortening [14]. The limitations are the lower temporal resolution and that no normal values for 3D TEE are available so far.

Image acquisition time to assess LV function and volume is equally between both $3 \mathrm{D}$ and $2 \mathrm{D}$. But, analysis of the 3D data

Table 1 Structure-specific 3D mode and clinical utility

\begin{tabular}{|c|c|c|c|}
\hline Structure & View & 3D Mode & Clinical utility [7] \\
\hline Mitral valve & ME $0-120^{\circ} \pm \mathrm{CFD}$ & Multiplane, Zoom, FV & Morphology (pathology), function, quantification-EROA and MVA \\
\hline Aortic valve & ME SAX and LAX views \pm CFD & Multiplane, Zoom, Live & $\begin{array}{l}\text { Valvular surgery and interventions esp. transcatheter } \\
\text { valvular replacements (TAVR) }\end{array}$ \\
\hline Tricuspid valve & $\operatorname{ME} 4 \mathrm{C}, 0^{\circ}-30^{\circ}, \mathrm{TG} \operatorname{SAX}\left(40^{\circ}\right)$ & Multiplane, Zoom, Live & Unstudied (transcatheter procedures experimental) \\
\hline Pulmonary valve & $\mathrm{UE} 90^{\circ}, \mathrm{ME} 3 \mathrm{C} 120^{\circ} \pm \mathrm{CFD}$ & Multiplane, Zoom & Limited visualization with TEE \\
\hline IAS & ME $0^{\circ}$ view & Multiplane, Zoom, FV & Atrial septal defect, patent foramen ovale \\
\hline LV & ME $0^{\circ}-120^{\circ}$ views & Multiplane, FV & Volume, mass, EF, dysynchrony, deformation parameters \\
\hline $\mathrm{RV}$ & ME $0^{\circ}-120^{\circ}$ & Multiplane, FV & Volume, EF \\
\hline LAA & $\operatorname{ME~} 0^{\circ}, 45^{\circ}, 60^{\circ}, 90^{\circ}$ & Multiplane, Live, Zoom & Thrombus \\
\hline
\end{tabular}

Modified from [14]

$C F D$ color flow Doppler, $E R O A$ effective orifice area, $E F$ ejection fraction, $I A S$ interatrial septum, $4 C$ four chamber, $F V$ full volume, $L A X$ long axis, $M E$ midesophageal, $M V A$ mitral valve area, $S A X$ short axis, TEE transesophageal echocardiography, $T G$ transgastric 
set takes on average 2 min longer [17]. In addition, a special software package for 3D analysis, not automatically included when buying a $3 \mathrm{D}$ system, is required.

Especially advantageous in the perioperative setting, nearly all left ventricular dimensions can be measured based on a single FV data set when using 3D TEE. This may be important in cardiac surgery when the patient is either unstable or there is not enough time for a comprehensive 2D TEE examination [18]. Multislice imaging of the ventricle can be performed, and regional wall motion and function can be assessed using this single data set.

\section{Right Ventricle Volume and Function}

The complex geometry of the right ventricle makes it impossible to evaluate right ventricular ejection fraction (EF) using 2D TEE. For experienced echocardiographers with access to special software, it is recommended to use 3D-derived right ventricular EF [14]. Good accuracy and correlation for 3D right ventricular function in cardiac surgical patients with preoperative 3D transthoracic echocardiography examinations have been shown in the perioperative setting [19, 20]. The main limitations are the need for special software and image quality.

\section{Mitral Valve}

The non-planar anatomy of the mitral valve (MV) complex lends itself to $3 \mathrm{D}$ assessment. It is more intuitive to assess the MV using 3D echocardiography than to reconstruct this threedimensional structure using multiple 2D views [21, 22]. The MV can be displayed from the left atrial side and the left ventricle side using one 3D echocardiography image or data set $[7 \bullet \bullet$. Assessment of the mechanism of MV pathology can be done accurately $[23,24]$; and 3D TEE is especially superior to $2 \mathrm{D}$ TEE in viewing complex MV anatomy and determining pathology $[25 \bullet \bullet, 26]$.

Three-dimensional MV assessment especially for MV repair surgery can be used to predict the probability of successful MV repair and to assess the result after repair [6••, 27], both leading to optimal outcomes after surgery [28].

\section{Quantification of Mitral Valve Disease (Mitral Regurgitation and Mitral Stenosis)}

Color flow Doppler in FV mode can be used to measure the $\mathrm{VC}$ area (VCA) and the proximal isovelocity surface area (PISA) by planimetry [24••, 29], overcoming the caveats of flow-derived calculation in 2D TEE [30]. The most important limitations are the temporal and spatial resolutions and cumbersome offline analysis [24••].

Three-dimensional TEE is remarkable in its ability to assess commissural fusion [31] in rheumatic MV disease, and its measurement of the MV area is as accurate as with 2D TEE [32].

\section{Localization of Paravalvular Leakage}

Paravalvular leaks can be exactly located [33] and quantified [34].

\section{Aortic Valve}

Three-dimensional echocardiography can accurately display the anatomy of the aortic valve in most patients and simplify communication between physicians [35], but in some patients, the imaging is limited due to acoustic shadowing [8] and echo dropout especially in degenerative calcified aortic cusps [9••].

Subvalvular aortic membranous structures as well as dynamic left ventricular outflow tract (LVOT) obstruction can be diagnosed by 3D echocardiography [36], but low temporal resolution may miss the dynamic obstruction [37].

Assessment of left ventricular outflow area with 3D echocardiography has been shown to correlate better with multislice computed tomography (MSCT) compared to 2D echocardiography $[38,39]$ and is, therefore, recommended [40••]. Sizing of transcatheter (transcatheter valvular replacements (TAVR)) valves is discussed in the section on transcatheter procedures/interventional procedures.

Pathology can be assessed by $2 \mathrm{D}$ and additionally by $3 \mathrm{D}$ TEE before aortic root, valve-sparing aortic, or valve repair surgery [24••, 41].

\section{Quantification of Aortic Valve Disease (Aortic Regurgitation and Aortic Stenosis)}

In aortic stenosis, 3D TEE can be used to measure left ventricular outflow area and diameter [40••]. Semi-automated 3D measurements seem to be better as compared to direct planimetry [42]. The underestimation of the LVOT area in the $2 \mathrm{D}$ method may lead to overestimation of aortic stenosis. Therefore, 3D echocardiography may have an impact on surgical decision making in these cases [39, 43]. Recent recommendations suggest the use of 3D TEE or MSCT to measure the LVOT and to use this measurement in the quantification of aortic valve area [40••].

The use of 3D color flow is not recommended in quantification of aortic regurgitation due to the low temporal and spatial resolutions, except in patients with multiple jets [41]. In these patients, overestimations or underestimations of the jet size may occur.

\section{Tricuspid Valve}

The assessment of the tricuspid valve (TV) and its pathology has to include assessment of the right ventricle and right 
atrium. Three-dimensional echocardiography allows accurate evaluation of right ventricle function and volumetric assessment with specialized and costly software.

Using biplane 3D TEE mode at different levels of the esophagus and stomach makes it possible to accurately identify the different leaflets [44•].

Color flow Doppler (CFD) can be added to the FV set to more accurately assess insufficiency or stenosis of the valve $[23,44]$. Vena contracta area (VCA), especially in the presence of multiple jets, can be assessed by 3D color Doppler echocardiography $[24 \cdot \bullet]$. Nevertheless, over- or underestimation may occur with inadequate spatial resolution. The threedimensional proximal isovelocity surface area (PISA) method in functional tricuspid regurgitation is clinically feasible and more correct than the 2D PISA method [45]. Planimetry of the tricuspid annulus can be done accurately and can detect eccentric dilatation in the presence of severe tricuspid regurgitation [46].

The greater distance between the probe and the TV and the non-perpendicular alignment make imaging of the TV by RT 3D TEE difficult [24・•].

\section{Pulmonary Valve}

The thin leaflets of the pulmonary valve (PV) are situated in the far field from the TEE probe, and an echo dropout artifact is frequently encountered. Therefore, RT 3D TEE has no advantage over 2D TEE in the clinical evaluation of the PV.

\section{Atria}

Three-dimensional echocardiography is more accurate in measuring left atrial (LA) and right atrial (RA) volumes and compares well with CMRI measurements [14]. Using RT 3D TEE, the atria cannot be fully measured due to the close proximity of the TEE probe to the atria. However, nearby structures can be visualized anatomically in detail (i.e., pulmonary vein ostia into the LA, vena cavae ostia, coronary sinus ostium, crista terminalis, eustachian valve, and fossa ovalis in the RA) [47].

\section{Great Vessels}

Three-dimensional TEE is more sensitive and detects cases of aortic aneurysms and dissections that are missed with 2D TEE. The dissection flap appears in 3D TEE as a tissue flap. The linear image of the dissection membrane can be mistaken for an artifact in a 2D examination. The involvement of the right coronary artery ostium in aortic dissections is more accurately diagnosed by 3D TEE [48].

\section{Interventional Cardiac Procedure}

The complexity of cardiac surgery and interventional procedures is increasing, and intraoperative and intraprocedural three-dimensional imaging is necessary to guide these $[49,50]$.

\section{Transcatheter Valvular Replacements}

While MSCT is the gold standard for sizing the aortic valve, 3D TEE is an accurate alternative in units where MSCT is not available or in situations where it is contraindicated [50-52]. The simultaneous multiplane mode can be used to correctly align the long axis of the aortic valve to measure the annulus accurately [52•]. Both simultaneous multiplane mode and RT 3D TEE are used during these procedures. With 3D TEE, annular distances to both coronary arteries can be measured using the multiplanar reconstruction (MPR) mode [50, 52, 53]. Assessing the position of the valve prosthesis on the balloon is better done with 3D TEE, because it can guide the correct positioning of the prosthesis in relation to the valve annulus and surrounding structures. The path of the guide wire through the LV and around the subvalvular apparatus of the MV is better visualized with RT 3D TEE [54]. Threedimensional TEE can also be used to assess postimplantation results, including the occurrence of intravalvular and paravalvular regurgitation [50,52].

\section{Paravalvular Leakage Diagnosis}

Live 3D zoom TEE images can be used to correctly localize paravalvular leaks. Care should be taken not to mistake echo dropout artifacts as paravalvular leaks. The size of the dehiscence can be determined by acquiring a FV set with CFD to confirm leakage and to measure its size [50].

\section{Transcatheter Mitral Valve Repair}

Guidance RT 3D TEE and simultaneous multiplane mode are strongly recommended for guidance of transcatheter edge to edge MV repair with the MitraClip ${ }^{\circledR}$ [55-58].

The Cardioband ${ }^{\circledR}$ direct annuloplasty MV repair is done with fluoroscopic and 2D/3D TEE guidance. Simultaneous biplane and 3D TEE are used for transseptal puncture and RT 3D TEE (wide-sector zoom) to assess anchoring position and final adjustment [59].

RT 3D TEE can be used in addition to 2D TEE and fluoroscopy to help with coronary sinus identification during the indirect coronary sinus annuloplasty technique [60].

Mitral stenosis can be treated by balloon valvotomy if found suitable for the procedure. Three-dimensional TEE can be used to assess the mitral commissural fusion to further determine suitability for this procedure. Three-dimensional 
TEE with its simultaneous biplane mode is used for correct transseptal puncture [57, 61].

\section{Left Atrial Interventions}

Intra-atrial Septum and Left Atrial Appendix Three-dimensional TEE is superior to 2D TEE in the spatial information that it provides to delineate the variant anatomy of a patent foramen ovale (PFO) and of atrial septal defects (ASD). The en-face image of the intra-atrial septum allows for spatial orientation and counting of defects. RT 3D TEE is also very suitable to guide the intervention. Offline MPR is useful for correct measurement of ASD size through precise alignment, can indicate rim deficiencies, and allows measurement relationships to important structures like the aorta [62•].

The sizing of the left atrial appendix (LAA) can be done by RT 3D TEE [63]. Two-dimensional TEE significantly underestimates the maximal LAA orifice compared to RT 3D TEE preclosure and during the procedure [64]. Additionally, RT 3D TEE is useful in ruling out a LAA thrombus [50].

\section{Infective Endocarditis and Cardiac/Aortic Sources of Embolism}

Infective endocarditis (IE) and its complications can readily be diagnosed and further clarified by the use of intraoperative 2D TEE. Three-dimensional TEE FV and real-time modes reveal pathology that is not readily seen with $2 \mathrm{D}$ echocardiography [65]. There is a good correlation between 3D TEE assessment of pathology and corresponding operative findings [66]. Morphology and size of valvular vegetations can be assessed, superior to 2D TEE [67].

Three-dimensional and multiplane imaging are suggested to confirm or exclude cardiac masses in the cardiac chambers [68].

Volumetric assessment of cardiac masses can be performed with 3D echocardiography, and further detailed assessment is possible: looking at attachment and detailed morphology [69].

Thromboembolism from the thoracic aorta and atheromatous plaque in the aortic arch should be visualized in $3 \mathrm{D}$ and multiplane images [70].

\section{Future Developments}

More complex percutaneous and transapical valve procedures are attempted and periprocedural 3D TEE guidance may also be useful in upcoming interventions [71-73].

Automated or semi-automated software for quantification of the MV, for example, is feasible [74, 75], but the benefit of this sophisticated software needs further investigation before routine clinical use.

Hybrid imaging techniques with the different imaging modalities merged and used at the same time will also improve the capabilities of 3D imaging [76]. Combined TEE and fluoroscopy merged/fused images may play a role in improved outcomes [28, 54].

Three-dimensional strain and strain rate as parameters of myocardial deformation for the assessment of the left ventricle, right ventricle, and left atrium will play an important role in chamber quantification in the future [77, 78•].

\section{Conclusion}

For the first time, RT 3D TEE allows for the anatomical display of most cardiac structures and pathologies, therefore facilitating improved communication between physicians. It additionally allows for more accurate quantification of some stenotic as well as regurgitant pathologies. Further development in 3D technology and the definition of normal values are the next steps to increase the clinical value of RT 3D TEE.

\section{Compliance with Ethical Standards}

Conflict of Interest Edwin Wilberforce Turton and Jörg Ender declare they have no conflict of interest.

Human and Animal Rights and Informed Consent This article does not contain any studies with human or animal subjects performed by any of the authors.

Open Access This article is distributed under the terms of the Creative Commons Attribution 4.0 International License (http:// creativecommons.org/licenses/by/4.0/), which permits unrestricted use, distribution, and reproduction in any medium, provided you give appropriate credit to the original author(s) and the source, provide a link to the Creative Commons license, and indicate if changes were made.

\section{References}

Papers of particular interest, published recently, have been highlighted as:

- Of importance

•- Of major importance

1. Matsumoto M, Oka Y, Lin YT, Strom J, Sonnenblick EH, Frater RW. Transesophageal echocardiography; for assessing ventricular performance. N Y State J Med. 1979;79(1):19-21.

2. Flachskampf FA, Wouters PF, Edvardsen T, Evangelista A, Habib G, Hoffman P, et al. Recommendations for transoesophageal echocardiography: EACVI update 2014. Eur Heart J Cardiovasc Imaging. 2014;15(4):353-65.

3. Pandian NG, Nanda NC, Schwartz SL, Fan P, Cao QL, Sanyal R, et al. Three-dimensional and four-dimensional transesophageal echocardiographic imaging of the heart and aorta in humans using a computed tomographic imaging probe. Echocardiography. 1992;9(6):677-87.

4. Fischer GW, Salgo IS, Adams DH. Real-time three-dimensional transesophageal echocardiography: the matrix revolution. J Cardiothorac Vasc Anesth. 2008;22(6):904-12. 
5. Pothineni KR, Inamdar V, Miller AP, Nanda NC, Bandarupalli N, Chaurasia $\mathrm{P}$, et al. Initial experience with live/real time threedimensional transesophageal echocardiography. Echocardiography. 2007;24(10):1099-104.

6.• Mahmood F, Matyal R. A quantitative approach to the intraoperative echocardiographic assessment of the mitral valve for repair. Anesth Analg. 2015;121(1):34-58. The importance of the intraoperative echocardiographer's role in assesings the mitral valve pathology and quantify it. Plays a role in decision making before and after the repair surgery

7.• Lang RM, Badano LP, Tsang W, Adams DH, Agricola E, Buck T, et al. EAE/ASE recommendations for image acquisition and display using three-dimensional echocardiography. J Am Soc Echocardiogr. 2012;25(1):3-46. The recommendations from the European and American experts, including the use of 3D TEE

8. Le HT, Hangiandreou N, Timmerman R, Rice MJ, Smith WB, Deitte L, et al. Imaging artifacts in echocardiography. Anesth Analg. 2016;122(3):633-46.

9.• Faletra FF, Ramamurthi A, Dequarti MC, Leo LA, Moccetti T, Pandian N. Artifacts in three-dimensional transesophageal echocardiography. J Am Soc Echocardiogr. 2014;27(5):453-62. Artifacts of three dimensional echocardiography, with the focus on specific artifacts during cardiac interventions

10. Mahmood F, Jeganathan J, Saraf R, Shahul S, Swaminathan M, Mackensen GB, et al. A practical approach to an intraoperative three-dimensional transesophageal echocardiography examination. J Cardiothorac Vasc Anesth. 2016;30(2):470-90. Review article on the quantitative and qualitative assessment using intraoperative 3D TEE

11. Saxena P, Malouf JF, Click R, Suri RM. 3D echocardiography in cardiac surgery. J Card Surg. 2014;29(1):51-4.

12.• Vegas A, Meineri M. Core review: three-dimensional transesophageal echocardiography is a major advance for intraoperative clinical management of patients undergoing cardiac surgery: a core review. Anesth Analg. 2010;110(6):1548-73. Initial comprehensive review article on this specific topic

13. Eibel S, Turton E, Mukherjee C, Bevilacqua C, Ender J. Feasibility of measurements of valve dimensions in en-face-3D transesophageal echocardiography. Int J Cardiovasc Imaging. 2017. doi:10. 1007/s10554-017-1141-z.

14.• Lang RM, Badano LP, Mor-Avi V, Afilalo J, Armstrong A, Ernande $\mathrm{L}$, et al. Recommendations for cardiac chamber quantification by echocardiography in adults: an update from the American Society of Echocardiography and the European Association of Cardiovascular Imaging. Eur Heart J Cardiovasc Imaging. 2015;16(3):233-70. Chamber quantification should include 3DE when applicable, and knowing it's advantages and limitations

15. Dorosz JL, Lezotte DC, Weitzenkamp DA, Allen LA, Salcedo EE. Performance of 3-dimensional echocardiography in measuring left ventricular volumes and ejection fraction: a systematic review and meta-analysis. J Am Coll Cardiol. 2012;59(20):1799-808.

16. Rigolli M, Anandabaskaran S, Christiansen JP, Whalley GA. Bias associated with left ventricular quantification by multimodality imaging: a systematic review and meta-analysis. Open Heart. 2016;3(1): $\mathrm{e} 000388$.

17. Meris A, Santambrogio L, Casso G, Mauri R, Engeler A, Cassina T. Intraoperative three-dimensional versus two-dimensional echocardiography for left ventricular assessment. Anesth Analg. 2014;118(4):711-20.

18. Ender A, Eibel S, Hasheminejad E, Scholz M, Kaisers UX, Mukherjee C, et al. Real-time 3 dimensional full volume data set : benefits in problem focused intraoperative transesophageal echocardiography. Anaesthesist. 2012;61(10):875-82.

19. Fusini L, Tamborini G, Gripari P, Maffessanti F, Mazzanti V, Muratori $\mathrm{M}$ et al. Feasibility of intraoperative three-dimensional transesophageal echocardiography in the evaluation of right ventricular volumes and function in patients undergoing cardiac surgery. J Am Soc Echocardiogr. 2011.

20. Karhausen J, Dudaryk R, Phillips-Bute B, Rivera JD, De LF, Milano CA, et al. Three-dimensional transesophageal echocardiography for perioperative right ventricular assessment. Ann Thorac Surg. 2012;94(2):468-74.

21. Salcedo EE, Quaife RA, Seres T, Carroll JD. A framework for systematic characterization of the mitral valve by real-time threedimensional transesophageal echocardiography. J Am Soc Echocardiogr. 2009;22(10): 1087-99.

22. Jain S, Malouf JF. Incremental value of 3-D transesophageal echocardiographic imaging of the mitral valve. Curr Cardiol Rep. 2014;16(1):439.

23.• Hahn RT, Abraham T, Adams MS, Bruce CJ, Glas KE, Lang RM, et al. Guidelines for performing a comprehensive transesophageal echocardiographic examination: recommendations from the American Society of Echocardiography and the Society of Cardiovascular Anesthesiologists. Anesth Analg. 2014;118(1):2168. Performing of 3D TEE is included in the guidelines

24.• Zoghbi WA, Adams D, Bonow RO, Enriquez-Sarano M, Foster E, Grayburn PA, et al. Recommendations for noninvasive evaluation of native valvular regurgitation: a report from the American Society of Echocardiography Developed in Collaboration with the Society for Cardiovascular Magnetic Resonance. J Am Soc Echocardiogr. 2017. Including both echocardiography and CMRI imaging to assess regurgitant valves, and the use of $3 \mathrm{DE}$ for both quantification and qualification are recommended.

25. Vegas A. Three-dimensional transesophageal echocardiography: principles and clinical applications. Ann Card Anaesth. 2016;19(Supplement):S35-43.

26. Tada H, Masuta E, Mori M, Tsubokawa T, Konno T, Hayashi K, et al. Perfect correspondence of mitral valve perforation using realtime 3-dimensional transesophageal echocardiography. J Am Coll Cardiol. 2012;59(21):1914.

27. Maslow A, Mahmood F, Poppas A, Singh A. Three-dimensional echocardiographic assessment of the repaired mitral valve. J Cardiothorac Vasc Anesth. 2014;28(1):11-7.

28. Wamil M, Bull S, Newton J. The modern role of transoesophageal echocardiography in the assessment of valvular pathologies. Echo Res Pract. 2017. doi:10.1530/ERP-16-0034.

29. Zeng X, Levine RA, Hua L, Morris EL, Kang Y, Flaherty M, et al. Diagnostic value of vena contracta area in the quantification of mitral regurgitation severity by color Doppler 3D echocardiography. Circ Cardiovasc Imaging. 2011;4(5):506-13.

30. Berdejo J, Shiota M, Mihara H, Itabashi Y, Utsunomiya H, Shiota T. Vena contracta analysis by color Doppler three-dimensional transesophageal echocardiography shows geometrical differences between prolapse and pseudoprolapse in eccentric mitral regurgitation. Echocardiography. 2017;34(5):683-89.

31. Schlosshan D, Aggarwal G, Mathur G, Allan R, Cranney G. Realtime 3D transesophageal echocardiography for the evaluation of rheumatic mitral stenosis. JACC Cardiovasc Imaging. 2011;4(6): $580-8$.

32. Dreyfus J, Brochet E, Lepage L, Attias D, Cueff C, Detaint D, et al. Real-time 3D transoesophageal measurement of the mitral valve area in patients with mitral stenosis. Eur J Echocardiogr. 2011;12(10):750-5.

33. Garcia-Fernandez MA, Cortes M, Garcia-Robles JA, Gomez de Diego JJ, Perez-David E, Garcia E. Utility of real-time three-dimensional transesophageal echocardiography in evaluating the success of percutaneous transcatheter closure of mitral paravalvular leaks. J Am Soc Echocardiogr. 2010;23(1):26-32.

34. Arribas-Jimenez A, Rama-Merchan JC, Barreiro-Perez M, Merchan-Gomez S, Iscar-Galan A, Martin-Garcia A, et al. Utility of real-time 3-dimensional transesophageal echocardiography in 
the assessment of mitral paravalvular leak. Circ J. 2016;80(3):73844.

35. Kim H, Bergman R, Matyal R, Khabbaz KR, Mahmood F. Threedimensional echocardiography and en face views of the aortic valve: technical communication. J Cardiothorac Vasc Anesth. 2013;27(2):376-80.

36. Jiang L, Shakil O, Montealegre-Gallegos M, Jainandunsing JS, Matyal R, Wang A, et al. Systolic anterior motion of the mitral valve and three-dimensional echocardiography. J Cardiothorac Vasc Anesth. 2015;29(1):149-50.

37. Hymel BJ, Townsley MM. Echocardiographic assessment of systolic anterior motion of the mitral valve. Anesth Analg. 2014;118(6):1197-201.

38. Gaspar T, Adawi S, Sachner R, Asmer I, Ganaeem M, Rubinshtein $\mathrm{R}$, et al. Three-dimensional imaging of the left ventricular outflow tract: impact on aortic valve area estimation by the continuity equation. J Am Soc Echocardiogr. 2012;25(7):749-57.

39. Jainandunsing JS, Mahmood F, Matyal R, Shakil O, Hess PE, Lee J, et al. Impact of three-dimensional echocardiography on classification of the severity of aortic stenosis. Ann Thorac Surg. 2013;96(4): 1343-8.

40.• Baumgartner H, Hung J, Bermejo J, Chambers JB, Edvardsen T, Goldstein S, et al. Recommendations on the echocardiographic assessment of aortic valve stenosis: a focused update from the European Association of Cardiovascular Imaging and the American Society of Echocardiography. J Am Soc Echocardiogr. 2017;30(4):372-92. Updated Recommendations, the use of 3D TTE and 3D TEE are recommended in imaging and quantification of AS

41. Zoghbi WA, Adams D, Bonow RO, Enriquez-Sarano M, Foster E, Grayburn PA, et al. Recommendations for noninvasive evaluation of native valvular regurgitation: a report from the American Society of Echocardiography Developed in Collaboration with the Society for Cardiovascular Magnetic Resonance. J Am Soc Echocardiogr. 2017;30(4):303-71.

42. Khalique OK, Hamid NB, White JM, Bae DJ, Kodali SK, Nazif $\mathrm{TM}$, et al. Impact of methodologic differences in three-dimensional echocardiographic measurements of the aortic annulus compared with computed tomographic angiography before Transcatheter aortic valve replacement. J Am Soc Echocardiogr. 2017;30(4):414-21.

43. Jainandunsing JS, Shahul SS. Moderate-to-severe aortic stenosis and three-dimensional echocardiography. J Cardiothorac Vasc Anesth. 2013;27(1):190-1.

44. Hahn RT. State-of-the-art review of echocardiographic imaging in the evaluation and treatment of functional tricuspid regurgitation. Circ Cardiovasc Imaging. 2016;9(12). Defining the role of 3D TTE and TEE in evaluation of the Tricuspid valve.

45. Muraru D, Surkova E, Badano LP. Revisit of functional tricuspid regurgitation; current trends in the diagnosis and management. Korean Circ J. 2016;46(4):443-55.

46. Mahmood F, Kim H, Chaudary B, Bergman R, Matyal R, Gerstle J, et al. Tricuspid annular geometry: a three-dimensional transesophageal echocardiographic study. J Cardiothorac Vasc Anesth. 2013;27(4):639-46.

47. Faletra FF, Nucifora G, Ho SY. Imaging the atrial septum using real-time three-dimensional transesophageal echocardiography: technical tips, normal anatomy, and its role in transseptal puncture. J Am Soc Echocardiogr. 2011;24(6):593-9.

48. Joshi D, Bicer EI, Donmez C, Hsiung MC, Nanda NC, Sadat K, et al. Incremental value of live/real time three-dimensional transesophageal echocardiography over the two-dimensional technique in the assessment of aortic aneurysm and dissection. Echocardiography. 2012;29(5):620-30.

49. Mahmood F, Shernan SK. Perioperative transoesophageal echocardiography: current status and future directions. Heart. 2016;102(15):1159-67.
50. Zamorano JL, Badano LP, Bruce C, Chan KL, Goncalves A, Hahn RT, et al. EAE/ASE recommendations for the use of echocardiography in new transcatheter interventions for valvular heart disease. J Am Soc Echocardiogr. 2011;24(9):937-65.

51. Zamorano J, Pardo A. 3D-ECHO for TAVI: two arrows, just in case. Eur Heart J Cardiovasc Imaging. 2016;17(1):9-10.

52. Hahn RT, Little SH, Monaghan MJ, Kodali SK, Williams M, Leon $\mathrm{MB}$, et al. Recommendations for comprehensive intraprocedural echocardiographic imaging during TAVR. JACC Cardiovasc Imaging. 2015;8(3):261-87. 3DE to size valves, identify landing zone and quantify paravalvular leaks

53. Patel PA, Gutsche JT, Vernick WJ, Giri JS, Ghadimi K, Weiss SJ, et al. The functional aortic annulus in the 3D era: focus on transcatheter aortic valve replacement for the perioperative echocardiographer. J Cardiothorac Vasc Anesth. 2015;29(1):240-5.

54. Perk G, Kronzon I. Interventional echocardiography in structural heart disease. Curr Cardiol Rep. 2013;15(3):338.

55. Debonnaire P, Delgado V, Bax JJ, Marsan NA. Tools \& techniques-clinical: 3D transoesophageal echocardiography for selecting and guiding in percutaneous mitral valve repair using MitraClip. EuroIntervention. 2014;10(7):884-6.

56. Guarracino F, Baldassarri R, Ferro B, Giannini C, Bertini P, Petronio AS, et al. Transesophageal echocardiography during MitraClip(R) procedure. Anesth Analg. 2014;118(6):1188-96. Increased value of 3D TEE in guiding this procedure

57. Cavalcante JL, Rodriguez LL, Kapadia S, Tuzcu EM, Stewart WJ. Role of echocardiography in percutaneous mitral valve interventions. JACC Cardiovasc Imaging. 2012;5(7):733-46.

58. Wunderlich NC, Beigel R, Siegel RJ. The role of echocardiography during mitral valve percutaneous interventions. Cardiol Clin. 2013;31(2):237-70.

59. Maisano F, Taramasso M, Nickenig G, Hammerstingl C, Vahanian A, Messika-Zeitoun D, et al. Cardioband, a transcatheter surgicallike direct mitral valve annuloplasty system: early results of the feasibility trial. Eur Heart J. 2016;37(10):817-25.

60. Mahmoud HM, Al-Ghamdi MA, Ghabashi AE. Real time threedimensional transesophageal echocardiography guided coronary sinus cannulation during CARILLON mitral annuloplasty device therapy for a patient with chronic severe mitral regurgitation. Echocardiography. 2015;32(1):181-3.

61. Perk G, Lang RM, Garcia-Fernandez MA, Lodato J, Sugeng L, Lopez J, et al. Use of real time three-dimensional transesophageal echocardiography in intracardiac catheter based interventions. J Am Soc Echocardiogr. 2009;22(8):865-82.

62. Silvestry FE, Cohen MS, Armsby LB, Burkule NJ, Fleishman CE, Hijazi ZM, et al. Guidelines for the echocardiographic assessment of atrial septal defect and patent foramen ovale: from the American Society of Echocardiography and Society for Cardiac Angiography and Interventions. J Am Soc Echocardiogr. 2015;28(8):910-58. Includes 3D TEE protocol for PFO and ASD assessment

63. Yosefy C, Laish-Farkash A, Azhibekov Y, Khalameizer V, Brodkin B, Katz A. A new method for direct three-dimensional measurement of left atrial appendage dimensions during transesophageal echocardiography. Echocardiography. 2016;33(1):69-76.

64. Goebel B, Wieg S, Hamadanchi A, Otto S, Jung C, Kretzschmar D, et al. Interventional left atrial appendage occlusion: added value of 3D transesophageal echocardiography for device sizing. Int $\mathrm{J}$ Cardiovasc Imaging. 2016;32(9):1363-70.

65. Vrettou AR, Zacharoulis A, Lerakis S, Kremastinos DT. Revealing infective endocarditis complications by echocardiography: the value of real-time 3D transesophageal echocardiography. Hell J Cardiol. 2013;54(2):147-9.

66. Yong MS, Saxena P, Killu AM, Coffey S, Burkhart HM, Wan SH, et al. The preoperative evaluation of infective endocarditis via 3 dimensional transesophageal echocardiography. Tex Heart Inst J. 2015;42(4):372-6. 
67. Berdejo J, Shibayama K, Harada K, Tanaka J, Mihara H, Gurudevan SV, et al. Evaluation of vegetation size and its relationship with embolism in infective endocarditis: a real-time 3-dimensional transesophageal echocardiography study. Circ Cardiovasc Imaging. 2014;7(1):149-54.

68. Mankad R, Herrmann J. Cardiac tumors: echo assessment. Echo Res Pract. 2016;3(4):R65-77.

69. Zaragoza-Macias E, Chen MA, Gill EA. Real time threedimensional echocardiography evaluation of intracardiac masses. Echocardiography. 2012;29(2):207-19.

70. Saric M, Armour AC, Arnaout MS, Chaudhry FA, Grimm RA, Kronzon I, et al. Guidelines for the use of echocardiography in the evaluation of a cardiac source of embolism. J Am Soc Echocardiogr. 2016;29(1):1-42.

71. Gafoor S, Petrescu OM, Lehr EJ, Puls C, Zhang M, Petersen JL II, et al. Percutaneous tricuspid valve regurgitation repair with the MitraClip device using an edge-to-edge bicuspidization technique. J Invasive Cardiol. 2017;29(3):E30-E6.

72. Schofer J, Tiburtius C, Hammerstingl C, Dickhaut PO, Witt J, Hansen L, et al. Transfemoral tricuspid valve repair using a percutaneous mitral valve repair system. J Am Coll Cardiol. 2016;67(7): 889-90.
73. Hammerstingl C, Schueler R, Malasa M, Werner N, Nickenig G. Transcatheter treatment of severe tricuspid regurgitation with the MitraClip system. Eur Heart J. 2016;37(10):849-53.

74. Mackensen GB, Swaminathan M, Mathew JP. PRO editorial: PRO: three-dimensional transesophageal echocardiography is a major advance for intraoperative clinical management of patients undergoing cardiac surgery. Anesth Analg. 2010;110(6):1574-8.

75. Noack T, Mukherjee C, Kiefer P, Emrich F, Vollroth M, Ionasec RI, et al. Four-dimensional modelling of the mitral valve by real-time 3D transoesophageal echocardiography: proof of concept. Interact Cardiovasc Thorac Surg. 2015;20(2):200-8.

76. Quaife RA, Salcedo EE, Carroll JD. Procedural guidance using advance imaging techniques for percutaneous edge-to-edge mitral valve repair. Curr Cardiol Rep. 2014;16(2):452.

77. Howard-Quijano K, Salem A, Barkulis C, Mazor E, Scovotti JC, Ho JK, et al. Preoperative three-dimensional strain imaging identifies reduction in left ventricular function and predicts outcomes after cardiac surgery. Anesth Analg. 2017;124(2):419-28.

78. Skubas NJ, London MJ. We should train for strain. Anesth Analg. 2017;124(2):386-7. May be used intraoperatively in the future 